\title{
Artificial Neural Network Simulation of Cyclone Pressure Drop: Selection of the Best Activation Function in Iraq
}

\author{
Selami Demir*, Aykut Karadeniz, Neslihan Manav Demir \\ Yıldız Technical University, Faculty of Civil Engineering, Environmental Engineering Department, \\ 34220, Esenler, Istanbul, Turkey
}

Received: 8 February 2016

Accepted: 26 April 2016

\begin{abstract}
A total of 162 cyclones with distinct geometries were used to obtain experimental pressure drops at six different inlet velocities between 10 and $24 \mathrm{~m} / \mathrm{s}$. Pressure drops were measured between 84 and 2,045 Pa. Pressure drop coefficients were calculated by the well-known formulation of a cyclone pressure drop. The values ranged between 1.09 and 9.07, with an average of value of 3.76. A backpropagation neural network algorithm was implemented in Visual Basic for Applications with nine built-in activation of linear, rectified linear, sigmoid, hyperbolic tangent, arctangent, Gaussian, Elliot, sinusoid, and sinc functions to test their ability to satisfactorily explain the complex relationship between cyclone geometry and the pressure drop coefficient. The neural network was run 25 times for each activation function with randomly selected $70 \%$ of data set as the ratios of inlet height, cylinder height, cone height, vortex finder diameter, and vortex finder length-to-body diameter being the independent variables, and the pressure drop coefficient being the dependent variable. Neural network results showed that sigmoid was the one activation function that explains the complex relationship between cyclone geometry and pressure drop coefficient with an average mean square error (MSE) of 0.00085 . The coefficients of determination between measured and predicted values of pressure drop coefficient were over 0.99. Also, the percent residuals from sigmoid activation function concentrated around the mean value of zero, with very small standard deviation.
\end{abstract}

Keywords: artificial neural network, cyclones, pressure drop, activation function

\section{Introduction}

Cyclone separators have been widely used for more than 100 years for separating particulate matter (PM) from industrial gas streams. Currently, the most common use of cyclones concentrates on flue gas control while they are also employed for recovering materials in powdered

*e-mail: seldemir@yildiz.edu.tr production facilities [1]. Cyclones for both purposes offer the advantages of simple construction and installation. Since they do not include any moving parts, they require less maintenance. Besides, cyclones can adapt to extreme operating conditions such as high temperatures and pressures, high PM loads, and corrosive gases [2-3].

Cyclones employ centrifugal force for removing PM from gas streams. The gas stream enters the cyclone at a high velocity from the top, usually through a rectangular 
tangential inlet structure. Various ranges of inlet velocities have been reported between $6-15 \mathrm{~m} / \mathrm{sn}$ and $15-25 \mathrm{~m} / \mathrm{sn}$ [4]. The cyclindirical body of the cyclone forces the gas stream into a rotating motion, resulting in the creation of a centrifugal force. Being heavier than the gaseous molecules, particulate matter moves outward to the cyclone wall, where they are collected, and moves downward in a cyclone wall-gas stream boundary. A conical part attached to the bottom of the cylinder is responsible for diverting gas stream upward to the vortex finder tube, through which clean gas leaves the cyclone. The particulate matter, on the other hand, does not change direction and falls to the bottom of the conical part, where a dust bin is employed to collect particulate matter.

Cyclone performance is defined by two operating parameters, namely pressure drop and collection efficiency. These parameters are intimately related to each other, and usually collection efficiency increases with increasing pressure drop until such a point where re-entrainment of particles occurs. Being the most widely used particulate collection device, a great number of attempts have been made to accurately estimate pressure drop and collection efficiencies, examples of which include Starimand [5], Casal and Martinez-Benet [6], Chen and Shi [7], and Demir [8]. Most of the previous studies have focused on developing some regression curves to estimate cyclone pressure drop. Since pressure drop is directly related to operating cost of the cyclone and is mainly a function of cyclone geometry, a great number of researchers have focused on optimizing cyclone geometry for reducing the pressure drop, and several standard cyclone geometries are currently available - one of which is the well-known Stairmand high-efficiency type.

Although computational fluid dynamics (CFD) applications have become very popular for simulating pressure and velocity fields in cyclone separators [2-3, 9-16], these models require high computational capacity and time for accurate estimations of operating parameters. In contrast to these deterministic models that are capable of explaining compex phenomena taking place in cyclones, another approach for modeling complex phenomena is a stochastic modeling tool called artificial neural networks (ANN).

A neural network is a modeling tool for highly complicated and nonlinear systems without understanding the nature of the phenomenon [17-18]. A neural network is a group of processing elements called neurons. Neurons are arranged in successive layers that are connected together by linear combinations of weights. Each neuron is defined by an activation function that converts the input signal into an output signal. The most commonly used type of ANN is backpropagation neural networks (BPNNs). Although ANNs have become very popular in recent years, their use for cyclone modeling is limited.

The aim of this study is to apply a neural network approach with backpropagation to estimate cyclone pressure drop. The neural network is used along with several activation functions to determine the best function for representing cyclone pressure drop.

\section{Material and Methods}

\section{Experimental Setup}

An experimental setup was built in the Air Pollution Research Laboratory of Yildı Technical University for investiging pressure drop in cyclones. The setup was composed of an air blower, an inlet channel, and a cyclone separator [19] (a schematic representation of the experimental setup is shown in Fig. 1). The air flow was produced by a variable-speed radial fan with a maximum flowrate of $1,500 \mathrm{~m}^{3} / \mathrm{h}$ and a maximum pressure of $1 \mathrm{~m}$ water column. The inlet channel is equipped with an orifice meter (ISO 5167-2:2003), a flow-control valve, and a differential pressure transmitter (Honeywell DPTM 1000D) to measure and control the air flowrate. QA/QC procedures for flowrate measurement using an orifice meter are given in detail in related standard documentation.

The experimental setup allows for cyclones of various geometries and dimensions. A total of 162 cyclones were used in the experiments. All of the cyclones had a body diameter of $290 \mathrm{~mm}$. The cyclone dimensions are given in Table 1. Since the cyclones discharged to the atmosphere, the pressure drop was measured as the static pressure at the inlet. Pressure measurements were also performed at the extended part of the vortex finder and no significant differences were observed.

The pressure drop in cyclones is usually estimated as a fraction of the number of velocity heads $\left(N_{H}\right)$, which is also called pressure drop coefficient of the cyclone. The pressure drop in a cyclone is calculated as follows:

$$
\Delta P=\frac{1}{2} \rho_{g} V_{i}^{2} N_{H}
$$

...where $\Delta P$ is the pressure drop in $\mathrm{Pa}, \rho_{g}$ is the gas density in $\mathrm{kg} / \mathrm{m}^{3}, V_{i}$ is the inlet gas velocity in $\mathrm{m} / \mathrm{s}$, and $N_{H}$ is the pressure drop coefficient. Extensive research has shown that the pressure drop coefficient is mainly a function of cyclone geometry, although several papers have reported that pressure drop is also a function of other parameters such as solid loading [20-21], and that pressure drop
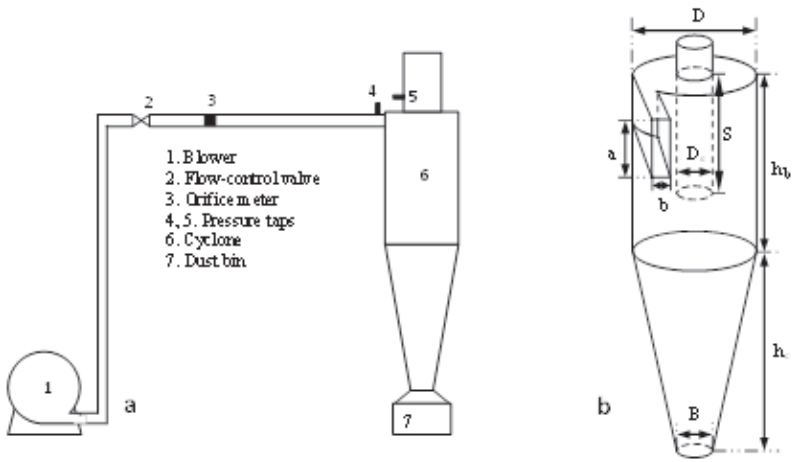

Fig. 1. Schematic representation of a) experimental setup, b) cyclone geometry. 
Table 1. Cyclone dimensions.

\begin{tabular}{|c|c|c|c|}
\hline Geometry & Notation & Dimension (mm) & Ratio \\
\hline Body diameter & $D$ & 290 & - \\
\hline Inlet height & $a$ & 116 & 0.40 \\
\hline & & 145 & 0.50 \\
\hline Inlet width & $b$ & 58 & 0.20 \\
\hline Cylinder height & $h_{b}$ & 435 & 1.50 \\
\hline & & 580 & 2.00 \\
\hline & & 725 & 2.50 \\
\hline Cone height & $h_{c}$ & 580 & 2.00 \\
\hline & & 725 & 2.50 \\
\hline & & 870 & 3.00 \\
\hline Vortex finder length & $S$ & 145 & 0.50 \\
\hline & & 174 & 0.60 \\
\hline & & 203 & 0.70 \\
\hline Vortex finder diameter & $D$ & 116 & 0.40 \\
\hline & & 145 & 0.50 \\
\hline & & 174 & 0.60 \\
\hline & $B$ & 109 & 0.38 \\
\hline
\end{tabular}

coefficient decreases with increasing solids loading, which leads to reduced pressure drop. But none of these parameters were considered in this study; thus excluding these parameters from the model provides a safe approach to calculate pressure drop.

\section{Artificial Neural Network}

An Excel Visual Basic for Applications (VBA) code was implemented for BPNN. The input layer consists of five neurons, respectively, for $a / D, h_{b} / D, h_{c} / D, S / D$, and $D_{e} / D$. Pressure drop coefficient $\left(N_{H}\right)$ is the only neuron in the output layer. The number of hidden layers, the number

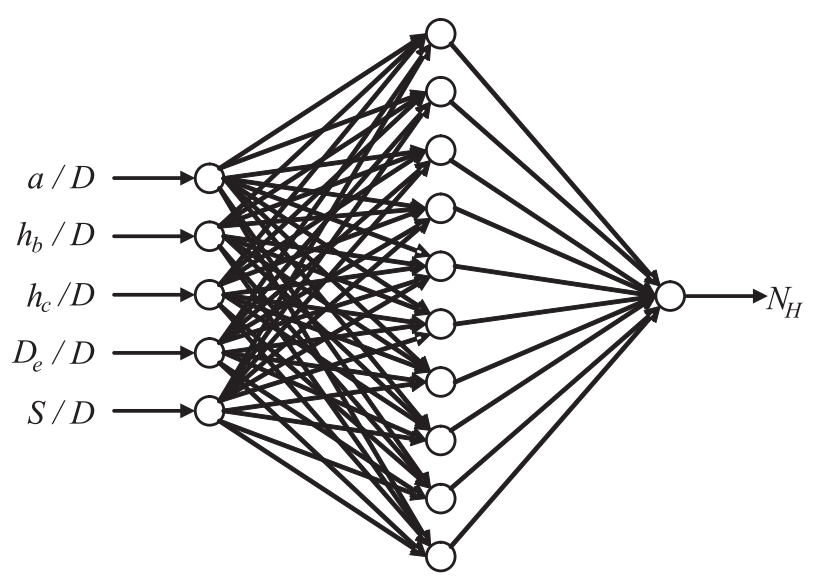

Fig. 2. Artificial neural network structure.
Table 2. Global options for ANN.

\begin{tabular}{|c|c|}
\hline Parameter & Value \\
\hline Number of input neurons & 5 \\
\hline Number of hidden layers & 1 \\
\hline Number of neurons in hidden layer & 10 \\
\hline Number of output neurons & 1 \\
\hline Percent of training data set (\%) & 70 \\
\hline Learning Rate & 0.75 \\
\hline Number of epochs & 5000 \\
\hline
\end{tabular}

of neurons in each hidden layer, and the activation function of each layer are user-defined parameters. Fig. 2 shows the general structure of the implemented ANN structure and Table 2 shows ANN global options.

\section{Activation Functions}

Activation function is a property of each neuron in hidden layers and the output layer. It determines how the neuron behaves, depending on the total input to the neuron. In this study, a number of well-known activation functions as well as some of those reported in Sibi et al. [22] were separately employed to determine their effects on ANN performance. The activation functions used are given in Table 3 along with their signal span and behaviour.

\section{Results and Discussion}

Each of 162 cyclones of distinct geometry and dimensions were run at six different inlet velocities between 10 and $24 \mathrm{~m} / \mathrm{s}$. Pressure drop ranged from 84 to $2,045 \mathrm{~Pa}$. For each cyclone geometry, pressure drop coefficient was calculated as the average of six values using Eq. (1). Pressure drop coefficients for each cyclone ranged between $1.09 \pm 0.19$ and $9.07 \pm 0.42$, with mean and median values of $3.76 \pm 2.14$ and 3.36, respectively.

The pressure drop coefficients were normalized prior to ANN run in accordance with the interval of span of activation function (Table 3). No normalizations were performed for linear and rectified linear activation functions. Normalized values of pressure drop coefficients were given to the implemented ANN software as target outputs. After each run, the ANN outputs were denormalized and compared with the measured values of pressure drop coefficient.

ANN software randomly selected $70 \%$ of the data set (113 data points) for each run. Thus, there was always the possibility of over- or under-learning. To avoid these extreme learning conditions, ANN software was run 25 times for each activation function and mean square error (MSE) for each were averaged for healthy comparison of the effects of activation function on ANN performance. 
Table 3. Behaviour of activation functions used.

\begin{tabular}{|c|c|c|c|}
\hline Function Name & Mathematical Expression & Derivative & Behaviour \\
\hline Linear* & $\begin{array}{c}y=0.01 x \\
\text { Span: }-\infty \leq y<\infty\end{array}$ & $\frac{d y}{d x}=0.01$ & \\
\hline Rectified linear* & $\begin{array}{c}y=\left\{\begin{array}{lll}0 & \Leftrightarrow & x<0 \\
0.01 x & \Leftrightarrow & x \geq 0\end{array}\right. \\
\text { Span: } 0 \leq y<\infty\end{array}$ & $\frac{d y}{d x}=\left\{\begin{array}{lll}0 & \Leftrightarrow & x<0 \\
0.01 & \Leftrightarrow & x \geq 0\end{array}\right.$ & 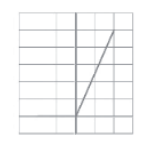 \\
\hline Logistic & $\begin{array}{c}y=\frac{1}{1+e^{-x}} \\
\text { Span: } 0<y<1\end{array}$ & $\frac{d y}{d x}=y(1-y)$ & $\int$ \\
\hline Hyperbolic tangent & $\begin{array}{c}y=\frac{e^{x}-e^{-x}}{e^{x}+e^{-x}} \\
\text { Span: }-1<y<1\end{array}$ & $\frac{d y}{d x}=1-y^{2}$ & \\
\hline Arctangent & $\begin{array}{c}y=\tan ^{-1}(x) \\
\text { Span: }-1 / 2 \pi<y<1 / 2 \pi\end{array}$ & $\frac{d y}{d x}=\frac{1}{1+x^{2}}$ & \\
\hline Gaussian & $\begin{array}{c}y=e^{-x^{2}} \\
\text { Span: } 0<y \leq 1\end{array}$ & $\frac{d y}{d x}=-2 x y$ & \\
\hline Elliot & $\begin{array}{c}y=\frac{0.5 x}{1+|x|}+0.5 \\
\text { Span: } 0<y<1\end{array}$ & $\frac{d y}{d x}=\frac{1}{2(1+|x|)^{2}}$ & 14 \\
\hline Sinusoid & $\begin{array}{c}y=\sin (x) \\
\text { Span: }-1 \leq y \leq 1\end{array}$ & $\frac{d y}{d x}=\operatorname{Cos}(x)$ & HA \\
\hline Sinc & $\begin{array}{l}y=\left\{\begin{array}{ccc}1 & \Leftrightarrow & x=0 \\
\frac{\sin (x)}{x} & \Leftrightarrow & x \neq 0\end{array}\right. \\
\text { Span: }-0.2172 \leq y \leq 1\end{array}$ & 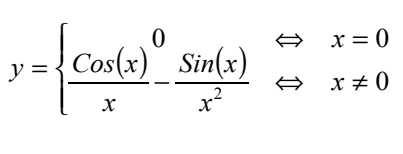 & $\sqrt{a}$ \\
\hline
\end{tabular}

$*$ These functions were used with a coefficient of 0.01 to prevent overflow in runtime.

Fig. 3 shows the changes in MSEs during the epochs for each activation function. MSEs are given as respective averages of calculated values in 25 ANN runs with the given activation function.

MSEs clearly show that hyperbolic tangent (Fig. 3d), arctangent (Fig. 3e), Gaussian (Fig. 3f), and sinusoid (Fig. $3 \mathrm{~h}$ ) activation functions were insufficient for effectively learning the training set pattern. The final values of MSEs at the end of 5,000 epochs were calculated as $0.86445,2.00878,0.08589$, and 0.91277 , respectively, for hyperbolic tangent, arctangent, Gaussian, and sinusoid activation functions. On the other hand, linear (Fig. 3a) and rectified linear (Fig. 3b) activation functions showed similar learning trends with final values of MSEs equal to 0.32125 and 0.12020 , respectively. Results were more representative compared to hyperbolic tangent, arctangent, Gaussian, and sinusoid when these functions were used as activation functions.

Mean square errors for sigmoid (Fig. 3c), Elliot (Fig. $3 \mathrm{~g}$ ), and sinc (Fig. 3i) functions were more satisfactory when compared to the others. Final MSEs were calculated as $0.00085,0.00213$, and 0.01129 , respectively, meaning that ANN with these functions can explain the nonlinear relationship between the cyclone geometry $\left(a, h_{b}, h_{c}, D_{e}\right.$, and $S$ ) and pressure drop coefficient $\left(N_{H}\right)$. Among these, the sigmoid function seems to be the one that explains the nonlinear relationship between input and output of the ANN (which are cyclone geometry and pressure drop coefficient, respectively).

Fig. 4 shows plots of measured pressure drop coefficients versus those calculated by BPNN. In the figure, the red diagonal line represents one-to-one values of measured and calculated pressure drop coefficients. The figure shows distribution of measured and calculated values (blue dots) and the regression line (black line) between these values with the regression equations. Clearly, hyperbolic tangent (Fig. 4d), arctangent (Fig. 4e), Gaussian (Fig. 4f), and sinusoid (Fig. 4h) activation functions were not suitable in their current form to explain the complex relationship between cyclone geometry and pressure drop coefficient. This was also evident with their calculated MSEs greater than 0.085 . The coefficient 

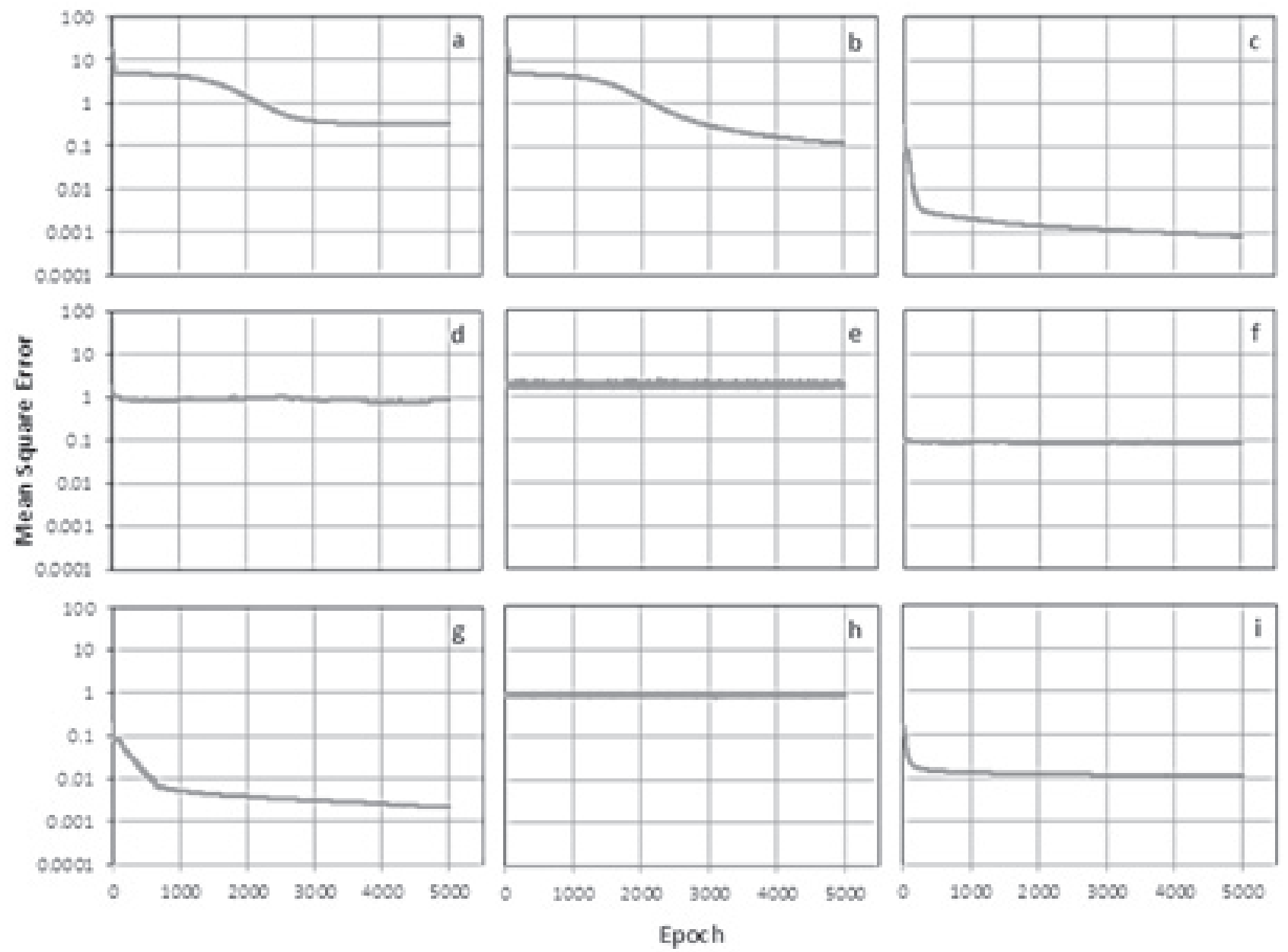

Fig. 3. Learning pattern of BPNN with a) linear, b) rectified linear, c) sigmoid, d) hyperbolic tangent, e) arctangent, f) Gaussian, g) Elliot, h) sinusoid, and i) sinc activation functions.

of determinations between measured and calculated pressure drop coefficients for these functions were $R^{2}=0.0412, R^{2}=0.0595, R^{2}=0.1024$, and $R^{2}=0.0000$, respectively, pointing out that there are no linear relationships between measured pressure drop coefficients and those calculated by ANN with these function - at all.

On the other hand, linear (Fig. 4a) and rectified linear (Fig. 4b) activation functions exhibited high determination coefficients with values $R^{2}=0.9300$ and $R^{2}=0.9758$, respectively. However, it is obvious from Figs. $4 \mathrm{a}$ and $4 \mathrm{~b}$ that these linear functions were insufficient to produce values in good agreement with the measured pressure drop coefficients. For good agreement, the slope and intercept of the regression line must be as close as 1 and 0 , respectively.

Elliot function, which resembles the shape of the curve $y=x^{-1}$ mirrored around the axis of $x=0$, better explains the complex relationship between cyclone geometry and pressure drop coefficient (Fig. 4g). The coefficient of determination was calculated as $R^{2}=0.9839$ for this function. Despite its high determination coefficient, on the other hand, the slope of the regression line (0.9394) is not close to unity very much, and visual inspection of Fig. $4 \mathrm{~g}$ clearly states that the results are not perfect. One good approximation to the measured pressure drop coefficients is from the sinc function (Fig. 4i). The coefficient of determination for this function was calculated as $R^{2}=0.9963$, while the slope of the regression line (0.9022) was even worse than that obtained with the Elliot function.

Fig. $4 \mathrm{c}$ clearly shows that sigmoid activation function was the best among the group for explaining the complex relationship between cyclone geometry and pressure drop coefficient. The coefficient of determination for sigmoid function was calculated as $R^{2}=0.9940$. Besides, the slope of the regression line was very close to unity, with a value of 0.9817 , while the intercept was 0.1354 . A closer look at Fig. 4 clearly reveals that ANN with sigmoid activation function was capable of explaining the complex relationship between cyclone geometry and pressure drop coefficient.

Frequency of occurences of percent residuals averaging more than 25 runs for each activation function as well as normal probability distributions of percent residuals are shown in Fig. 5. The column plots in the figure represent averages of frequencies of percent residuals over 25 runs, while the red curves represent normal probability distributions of percent residuals to be expected when the respective activation function is used. 

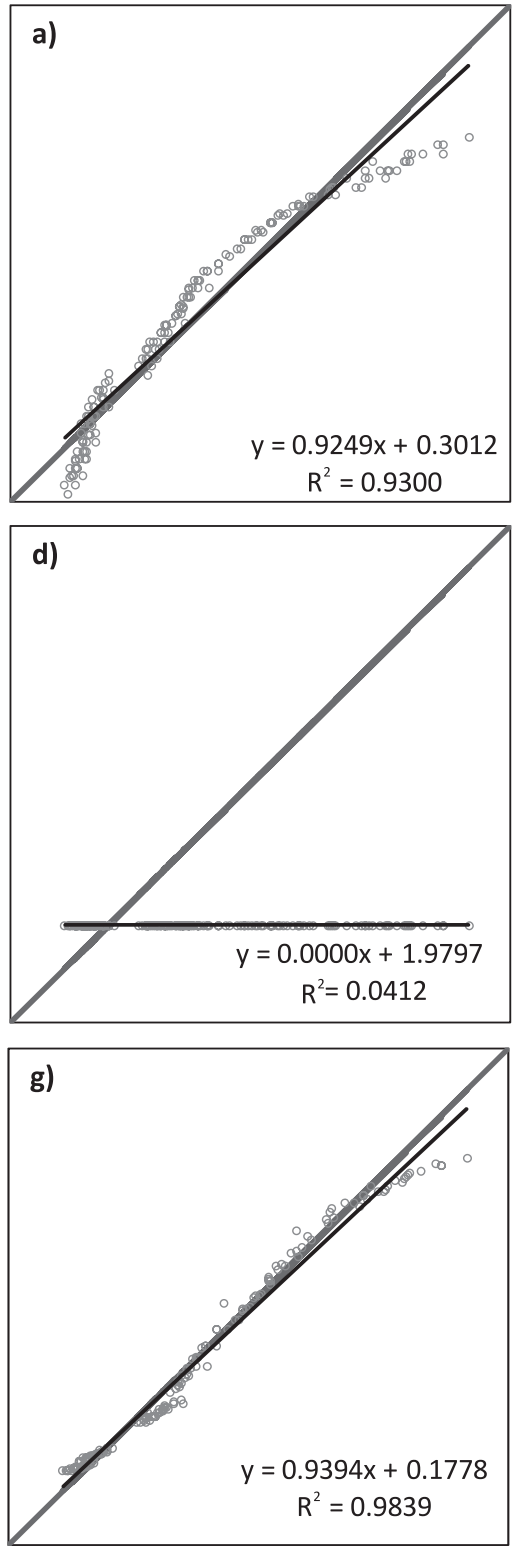
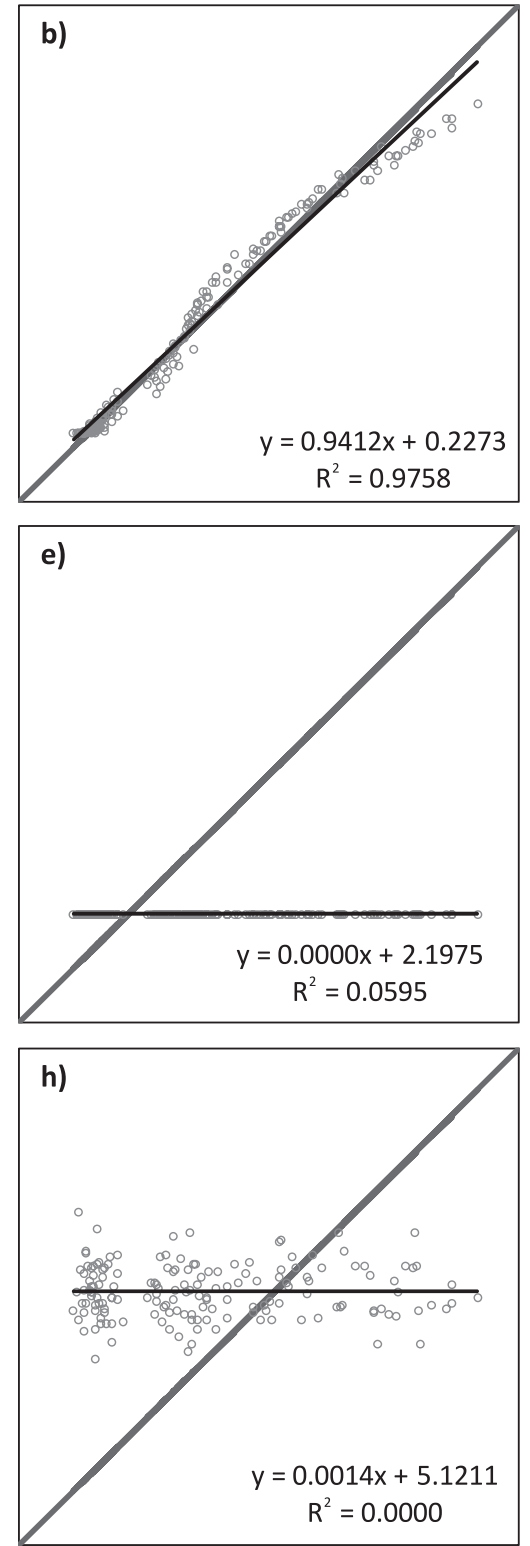
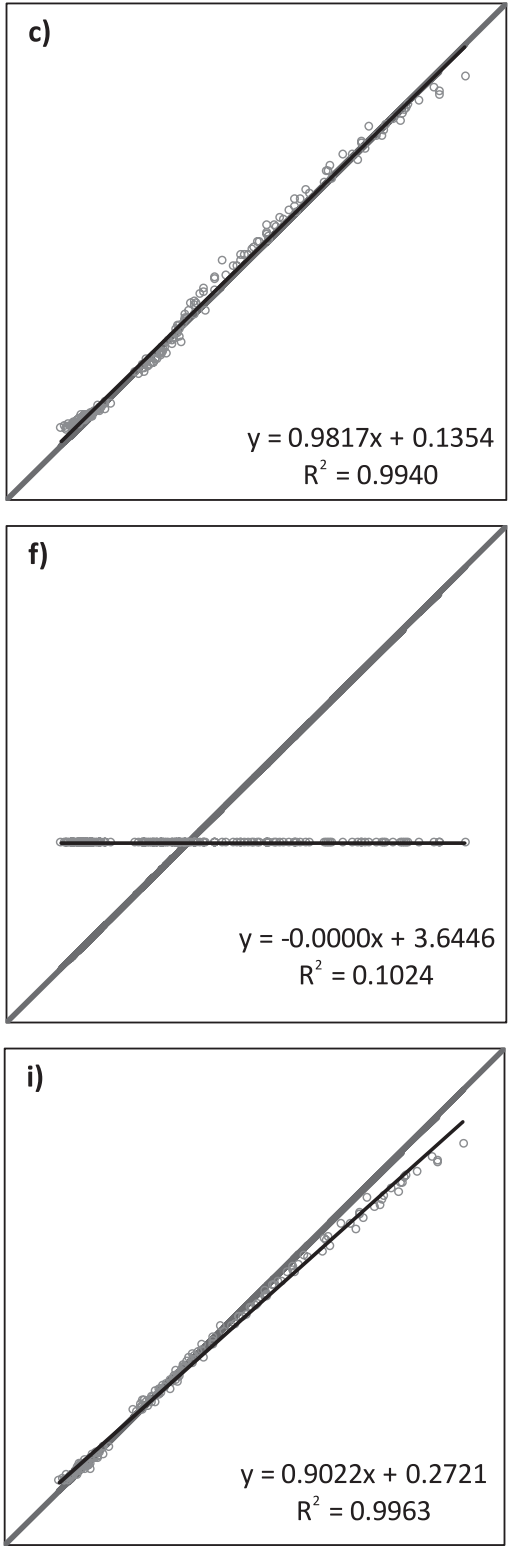

Fig. 4. Measured versus calculated pressure drop coefficients by BPNN with a) linear, b) rectified linear, c) sigmoid, d) hyperbolic tangent, e) arctangent, f) Gaussian, g) Elliot, h) sinusoid, and i) sinc activation functions.

Normal probabilities of percent residuals for hyperbolic tangent (Fig. 5d), arctangent (Fig. 5e), Gaussian (Fig. 5f), and sinusoid (Fig. 5h) functions clearly show that, with these functions' current forms, percent residuals between the predicted and measured values of pressure drop coefficients could reach $100 \%$ depending on the function used. The percent residuals observed for linear (Fig. 5a) and rectified linear (Fig. 5b) activation functions seem more-or-less normally distributed around the mean of zero. However, greater negative fluctuations were observed for linear activation function. Besides, Figs $4 \mathrm{a}$ and $4 \mathrm{~b}$ clearly show that these functions were unable to explain the nonlinear relationship between cyclone geometry and pressure drop coefficient. Elliot activation function (Fig. $5 \mathrm{~g}$ ), on the other hand, clearly caused a positive skewness in percent residuals. Besides, $\pm 40 \%$ fluctuation from the measured pressure drop coefficient was probable with this function. Another activation function, the sinc function (Fig. 5i), seems like a very good predictor of pressure drop coefficients. However, Fig. 4i clearly shows that the slope of the regression line between measured and predicted pressure drop coefficients was not as close to unity as that calculated for the sigmoid function. Percent residuals calculated with the sigmoid function (Fig. 5c) seems normally distributed around the mean value of zero. Besides, the expected frequency of $\pm 5.0 \%$ residuals was as high as 0.5 , meaning that most of the predicted values of pressure drop coefficient will fall in this range. The percent residuals shown in Fig. 5 support the discussion related with the findings in Fig. 4, and there is sufficient evidence to conclude that the sigmoid function is the one activation function for a BPNN that explains the complex 

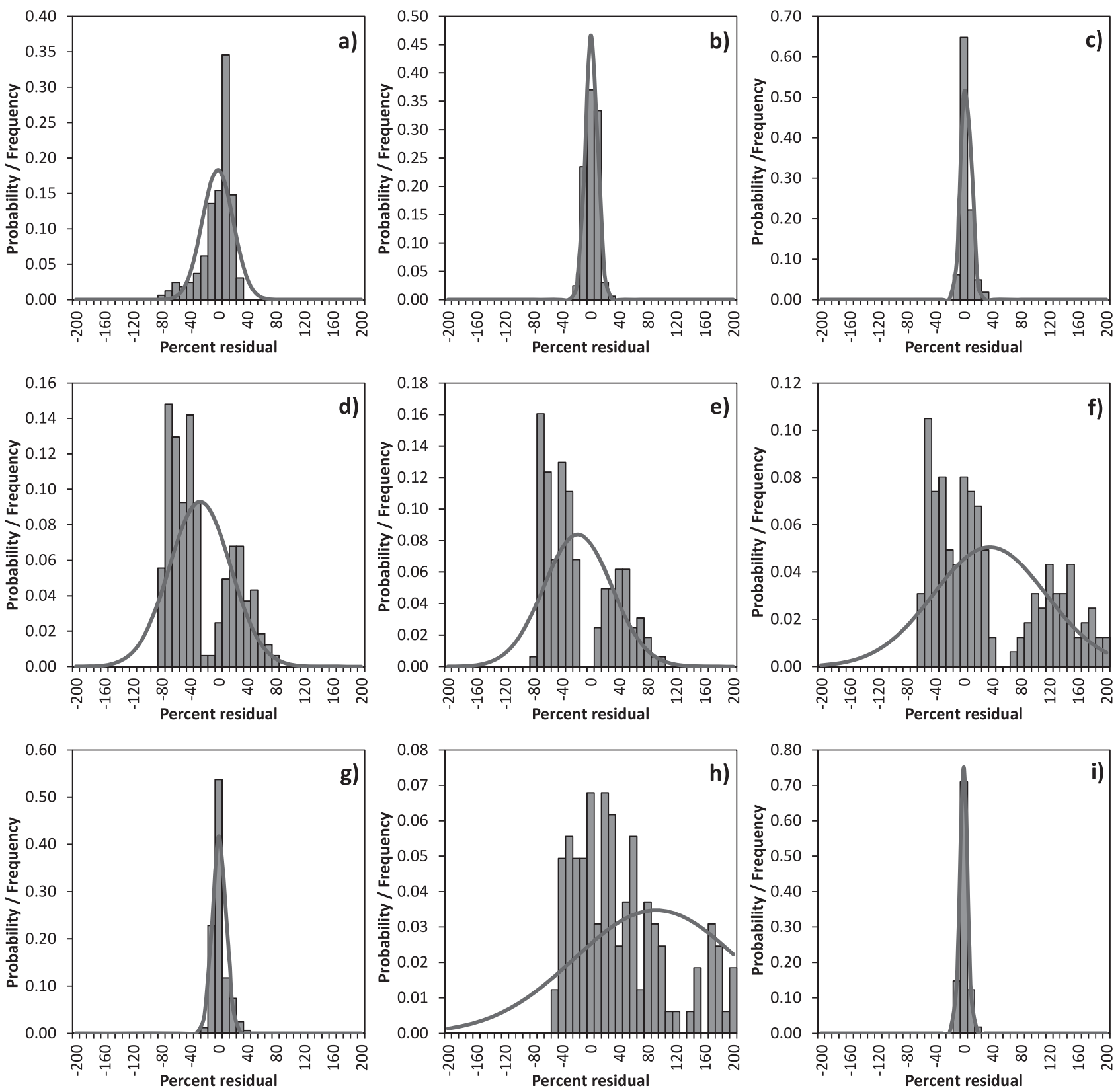

Fig. 5. Percent residuals and probability distributions by BPNN with a) linear, b) rectified linear, c) sigmoid, d) hyperbolic tangent, e) arctangent, f) Gaussian, g) Elliot, h) sinusoid, and i) sinc activation functions.

nonlinear relationship between cyclone geometry and pressure drop coefficient.

A total of 162 cyclones were used in this study. For each cyclone, pressure drop coefficient was calculated as the average of six measurement results. Twenty-five runs were performed during the ANN simulation, and Figs 3 and 4 are based on the averages of the results calculated in those runs. Thus, the measurement and simulation data formed two matrices. The former was six by 162 and the latter was 25 by 162 . One-way analysis of variance (ANOVA) was performed for each column of these two matrices to check whether the simulation results were in good agreement with the measurement results. The tests were performed at a confidence level of $95 \%$, with degrees of freedom $v_{1}=1$ and $v_{2}=29$ (six observations for measured and 25 for calculated values of pressure drop coefficient), and with the null hypothesis that the means of the measured and calculated pressure drop coefficients in each pair in the data set were equal. The critical value of $F$ was 4.18. The one-way ANOVA tests on 162 cyclones suggested accepting the null hypothesis in 106 out 162 , corresponding to $65.4 \%$ of the calculated pressure drop coefficients agreeing with the measured values.

\section{Conclusions}

A total of 162 cyclones of various geometries, all of them having the same body diameter of $290 \mathrm{~mm}$, were run at six different inlet velocities between 10 and $24 \mathrm{~m} / \mathrm{s}$. 
Maximum and minimum pressure drop were measured as 84 and 2,045 $\mathrm{Pa}$. Since most of the pressure drop models for cyclones were calculated using a pressure drop coefficient $\left(N_{H}\right.$, also called total number of velocity heads), six pressure drop coefficients for each cyclone were calculated and averaged.

It is well known that the pressure drop coefficient of a cyclone is a function of it geometry. Thus a cyclones' geomety (namely $a / D, h_{b} / D, h_{c} / D, D_{e} / D$, and $S / D$, along with average values of pressure drop coefficients) were used as input data set in a BPNN. A VBA code was implemented for the neural network algorithm. Cyclone geometry was used as independent variables and the pressure drop coefficient as the dependent variable. Nine activation functions were used with the neural network to test the performance of the most commonly used activation functions. A randomly selected $70 \%$ of the data set was used as the training data set. For each function, 25 runs were performed and results were averaged. The neuralnetwork-calculated pressure drop coefficients were compared with measured values for assessing activation function performances. Also, one-way ANOVA tests were performed between each measured and neural-networkcalculated results to numerically check whether calculated results are in good agreement with the measurements or not.

The following conclusions can be drawn from this study:

- Trigonometric functions (sinusoid and arctangent) as well as hyperbolic tangent and Gaussian activation functions were not suitable in their current forms for explaining the complex relationship between cyclone geometry and pressure drop coefficient.

- Although linear functions (linear and rectified linear) produced good results compared to trigonometric functions, their inefficiency for representing a nonlinear relationship limits their widespread use for cyclones.

- Nonlinear activation functions were better for explaining the relationship between cyclone geometry and pressure drop coefficient.

- The sigmoid activation function was capable of explaining the complex relationship for cyclones with coefficients of determination between measured and predicted pressure drop coefficients greater than 0.99 .

- It is the authors' belief that some of the activation functions such as hyperbolic tangent were insufficient for the purpose of modeling the cyclone pressure drop due only to their steep slopes when their inputs were close to zero. The performance of these functions must be further investigated by incorporating steepness coefficients to reduce the absolute value of their slopes and improve their suitability for cyclone pressure drop.

\section{Abbreviations}

$a=$ Inlet height of cyclone (mm)

$b=$ Inlet width of cyclone ( $\mathrm{mm})$

$B=$ Cone-tip diameter of cyclone (mm)
$\mathrm{BPNN}=$ Back propagation neural network

$D=$ Cyclone body diameter $(\mathrm{mm})$

$D_{e}=$ Vortex finder diameter of cyclone (mm)

$N_{H}^{e}=$ Number of velocity heads (dimensionless)

$h_{b}=$ Cylinder (body) height of cyclone (mm)

$h_{c}=$ Cone height of cyclone $(\mathrm{mm})$

MSE $=$ Mean square error

$S_{L}=$ Vortex finder length of cyclone ( $\mathrm{mm}$ )

$V_{i n}=$ Inlet velocity to the cyclone $(\mathrm{m} / \mathrm{s})$

$\Delta P=$ Pressure drop in cyclone $(\mathrm{Pa})$

$\rho=$ Gas density (kg/m.s.)

\section{Acknowledgements}

This research was supported by the Yildiz Technical University Scientific Research Projects Coordination Department (project No. 2012-05-02-KAP04).

\section{References}

1. COOPER C.D., ALLEY F.C. Air Pollution Control: A Design Approach, $4^{\text {th }}$ ed., Waveland Press, Inc., Illionis, USA, 135, 2011.

2. ELSAYED K., LACOR C. The effect of vortex finder diameter on cyclone separator performance and flow field. Proceedings of the 5th European Conference on Computational Fluid Dynamics (ECCOMAS CFD 2010), Portugal, 1, 2010.

3. ELSAYED K., LACOR C. Numerical modeling of the flow field and performance in cyclones of different cone-tip diameters. Comput. Fluids 51, 48, 2011.

4. HERRICK T.C., DAVIS W.T. Cyclones and Inertial Separators, in: W.T. Davis (Ed.), Air Pollution Engineering Manual, Air and Waste Management Association, John Wiley \& Sons, USA, 66, 2000.

5. STAIRMAND C.J. Pressure drop in cyclone separators. Eng. Lond. 16B, 409, 1949.

6. CASAL J., MARTINEZ-BENET J.M. A better way to calculate cyclone pressure drop. Chem. Eng. N.Y. 99, 100, 1983.

7. CHEN J., SHI M. A universal model to calculate cyclone pressure drop. Powder Technol. 171, 184, 2007.

8. DEMIR S. A practical model for estimating pressure drop in cyclone separators: An experimental study. Powder Technol. 268, 329, 2014.

9. KAYA F., KARAGÖZ I. Investigation into the suitability of turbulence models in swirling flows. Uludağ University Journal of Engineering and Archiecture Faculty 12, 85, 2007 [In Turkish].

10. RAOUFI A., SHAMS M., FARZANEH M., EBRAHIMI R. Numerical simulation and optimization of fluid flow in cyclone vortex finder. Chem. Eng. Process. 47, 128, 2008.

11. ELSAYED K., LACOR C. Optimization of cyclone separator geometry for minimum pressure drop using mathematical models and CFD simulations. Chem. Eng. Sci. 65, 6048, 2010.

12. ELSAYED K., LACOR C. The effect of cyclone inlet dimensions on the flow pattern and performance. Appl. Math. Model. 35, 1952, 2011.

13. ELSAYED K., LACOR C. Modeling, analysis and optimization of aircyclones using artifical neural network, 
response surface methodology and CFD simulation Approaches. Powder Technol. 212, 115, 2011.

14. ELSAYED K., LACOR C. The effect of the dust outlet geometry on the performance and hydrodynamics of gas cyclones. Comput. Fluids 68, 134, 2012.

15. BRAR L.S., SHARMA R.P., ELSAYED K. The effect of the cyclone length on the performance of stairmand highefficiency cyclone. Powder Technol. 286, 668, 2015.

16. BRAR L.S., SHARMA R.P. Effect of varying diameter on the performance of industrial scale gas cyclone dust separators. Materials Today: Proceedings 2, 3230, 2015.

17. ZHAO B., SU Y. Artificial neural network-based modeling of pressure drop coefficient for cyclone separators. Chem. Eng. Res. Des. 88 (5-6), 606, 2010.

18. ELSAYED K., LACOR C. Modeling and pareto optimization of gas cyclone separator performance using RBF type artificial neural networks and genetic algorithms. Powder Technol. 217, 84, 2012.
19. KARADENIZ A. Effect of modifications on stairmand high efficiency type cyclone geometry on particle collection efficiency and pressure drop. MSc Thesis, Graduate School of Natural and Applied Sciences, Y1ldı Technical University, Istanbul, 2015 [In Turkish].

20. MUCHELKNAUTZ E. The calculations for cyclone separators for gases. Chem. Ing. Tech. 44 (1-2), 63, 1972 [In German].

21. HOFFMANN A.C., VAN SANTEN A., ALLEN R.W.K. Effects of geometry and solid loading on the performance of gas cyclones. Powder Technol. 70, 1326, 1992.

22. SIBI P., JONES A.A., SIDDARTH P. Analysis of different activation functions using back propagation neural networks. Journal of Theoretical and Applied Information Technology 47 (3), 1264, 2013 
